\title{
Initial Guesses Generation for Fluorescence Intensity Distribution Analysis
}

Victor V. Skakun ${ }^{1,3}$, Eugene G. Novikov²,. Vladimir V. Apanasovich ${ }^{3}$, Hans Tanke ${ }^{4}$, Andre Deelder ${ }^{1}$, Oleg A. Mayboroda ${ }^{1}$

${ }^{1}$ Department of Parasitology, Leiden University Medical Centre, Leiden, The Netherlands

2 Service Bioinformatique, Institut CURIE, Paris, France

3 Department of Systems Analysis, Belarusian State University, Minsk, Belarus

${ }^{4}$ Department of Molecular Cell Biology, Leiden University Medical Centre, Leiden, The Netherlands 


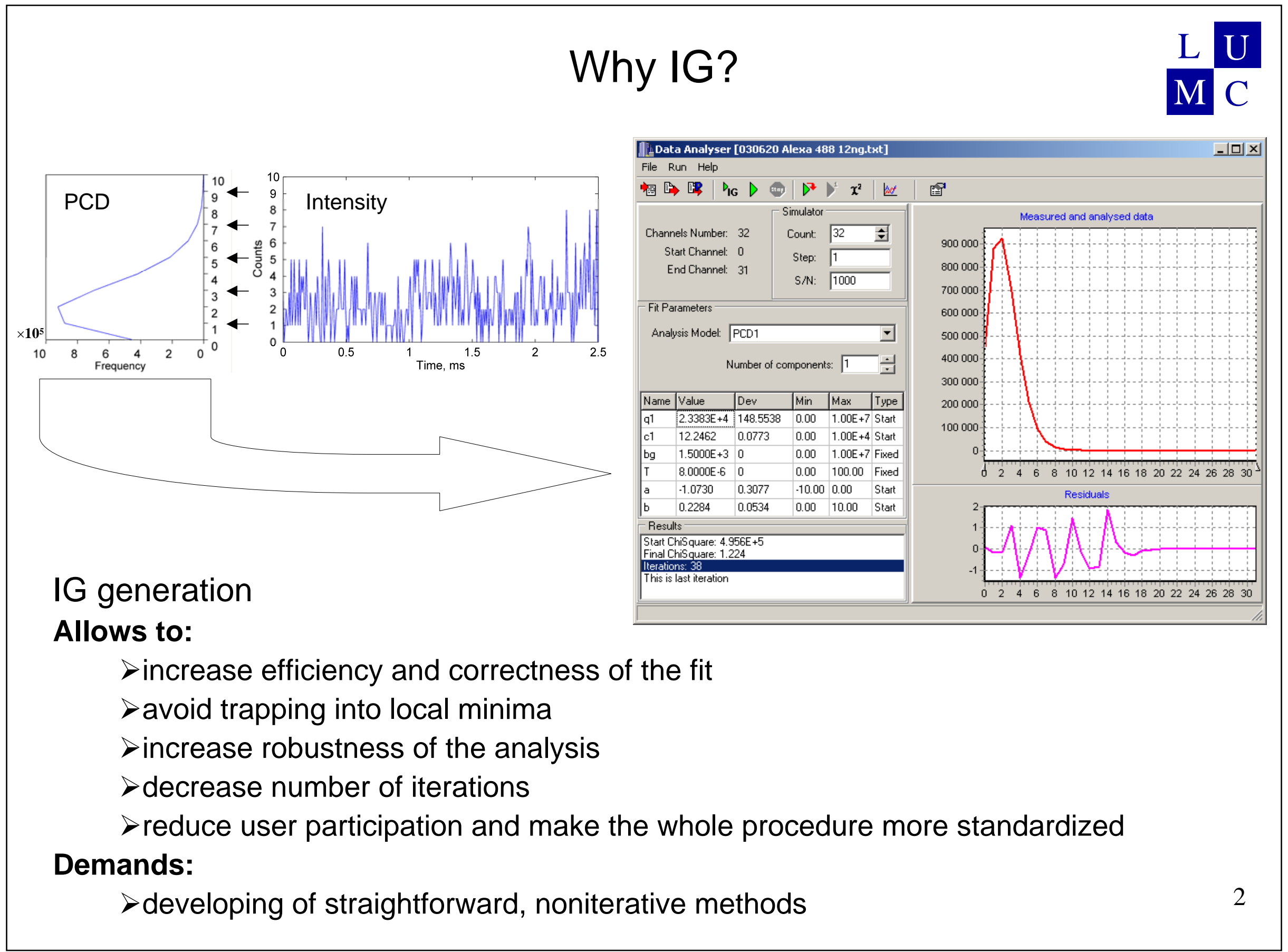




\section{Method of moments}

$M_{k}\left(\eta_{1}, \eta_{2}, \ldots, \eta_{m}\right)=\tilde{M}_{k}, \quad k=1,2, \ldots, m, \quad$ (1)

where $\eta_{1}, \eta_{2}, \ldots, \eta_{m}$ is a set of unknown parameters

$\tilde{M}_{k}=<n^{k}>=\sum_{n=1}^{N-1} n^{k} P^{*}(n)$

$P^{*}(\mathrm{n})$ is a probability to get $n$ photons within a counting time interval $T$

in application to factorial cumulants

$$
\begin{aligned}
& K_{k}\left(\eta_{1}, \eta_{2}, \ldots, \eta_{m}\right)=\tilde{K}_{k} \\
& K_{k}=\left.\frac{d^{k} \ln G(\xi)}{d \xi^{k}}\right|_{\xi=1} \text { (3) } \quad G(\xi)=\sum_{n=0}^{\infty} \xi^{n} P(n) \\
& \tilde{F}_{k}=<n(n-1) \ldots(n-k+1)>=\sum_{n=k}^{N-1} n(n-1) \ldots(n-k+1) P^{*}(n) \\
& \tilde{K}_{k}=\tilde{F}_{k}-\sum_{i=1}^{k-1}\left(\frac{k-1}{i}\right) \tilde{K}_{k-i} \tilde{F}_{i}, \quad \text { where }\left(\frac{k-1}{i}\right)=\frac{(k-1) !}{i !(k-i-1) !}
\end{aligned}
$$




\section{Historical background}

\section{Moment analysis of fluorescence fluctuations}

$$
\left\{\begin{array}{l}
<\Phi>=\chi_{1} \sum_{i} q_{i} c_{i} \\
<\Delta \Phi^{2}>=\chi_{2} \sum_{i} q_{i}^{2} c_{i} \\
<\Delta \Phi^{3}>=\chi_{3} \sum_{i} q_{i}^{3} c_{i} \\
<\Delta \Phi^{4}>-3<\Delta \Phi^{2}>^{2}=\chi_{4} \sum_{i} q_{i}^{4} c_{i} \\
\chi_{k}=\int B^{k}(r) d r
\end{array}\right.
$$

here $\Phi$ is the fluorescence intensity, $c_{i}$ is the number of molecules per observation volume, $q_{i}$ is the specific brightness expressed in cpm, $B(r)$ is a spatial brightness function, $i$ is the number of molecular species

Qian, H., and E.L. Elson. Biophys. J. 57, 1990

Qian, H., and E.L. Elson. PNAS 87, 1990

\section{Fluorescence Cumulant Analysis}

$$
K_{k}=\chi_{k} \sum_{i} c_{i} q_{i}^{k}
$$




\section{FIDA}

$$
\begin{aligned}
& G(\xi)=\sum_{n=0}^{\infty} \xi^{n} P(n) \quad(11) \quad P(n) \text { is photon counting distribution (PCD) } \\
& G(\xi)=\exp \left((\xi-1) \lambda T+\sum_{j} c_{j} \int_{V}\left\{\exp \left[(\xi-1) q_{j} T B(r)\right]-1\right\} d V\right)
\end{aligned}
$$

here $c_{j}$ is the mean number of molecules per observation volume, $q_{j}$ is the specific brightness expressed in cpmt, $V$ is the observation volume, $T$ is the counting time interval, $B(r)$ is brightness profile function which is the product excitation intensity and detection efficiency, $j$ is the number of molecular species and $\lambda$ is the mean background count rate of detector.

Evotec Biosystems AG. 1998. Int. Patent WO 98/16814.

Kask at al. PNAS 96, 1999.

$$
\frac{d V}{d x}=A_{0}\left(x+a x^{2}+b x^{3}\right), \quad x=\ln \left[B_{0} / B(r)\right], \quad B(r)=B_{0} e^{-x}
$$

where $a, b$ are instrumental parameters and $A_{0}, B_{0}$ can be calculated from system of normalization equations:

$$
\begin{aligned}
& \chi_{1}=\int_{V} B(r) d V=1, \\
& \chi_{2}=\int_{V} B^{2}(r) d V=1 .
\end{aligned}
$$

Finally $P(n)=F F T^{-1}\left(G\left(e^{i \varphi}\right)\right)$

Evotec Biosystems AG. 1998. Int. Patent WO 98/16814. 


\section{General system of equations for IG generation}

$$
\begin{aligned}
K_{k} & =\left.\frac{d^{k} \ln G(\xi)}{d \xi^{k}}\right|_{\xi=1} \\
K_{1} & =\left(\lambda+\sum_{j} c_{j} q_{j}\right) T \\
K_{2} & =\sum_{j} c_{j} q_{j}^{2} T^{2} \\
K_{k} & =\chi_{k} \sum_{j} c_{j} q_{j}^{k} T^{k}, \quad k=3,4, \ldots, \\
\chi_{k} & =\int_{0}^{\infty}\left(B_{0} e^{-x}\right)^{k} A_{0}\left(x+a x^{2}+b x^{3}\right) d x \\
B_{0} & =\frac{8(2 a+6 b+1)}{2 a+3 b+2}, \quad A_{0}=\frac{2 a+3 b+2}{8(2 a+6 b+1)^{2}}
\end{aligned}
$$




\section{IG for one component model}

\section{Basic system of equations:}

estimated parameters are $c, q, \lambda, a, b$

$$
\left\{\begin{array}{l}
K_{1}=(\lambda+c q) T \\
K_{2}=c q^{2} T^{2} \\
K_{3}=c q^{3} T^{3} \frac{64(2 a+6 b+1)(2 a+2 b+3)}{27(2 a+3 b+2)^{2}} \\
K_{4}=c q^{4} T^{4} \frac{4(2 a+6 b+1)^{2}(4 a+3 b+8)}{(2 a+3 b+2)^{3}} \\
K_{5}=c q^{5} T^{5} \frac{4096(2 a+6 b+1)^{3}(10 a+6 b+25)}{625(2 a+3 b+2)^{4}}
\end{array}\right.
$$

\section{Solution:}

$$
\begin{aligned}
& \left\{\begin{array}{l}
\frac{(10 a+6 b+25)(2 a+2 b+3)}{(4 a+3 b+8)^{2}}=\frac{16875 K_{5} K_{3}}{16384 K_{4}^{2}} \\
\frac{729(2 a+3 b+2)(4 a+3 b+8)}{(2 a+2 b+3)^{2}}=\frac{1024 K_{4} K_{2}}{729 K_{3}^{2}}
\end{array}\right. \\
& \lambda=K_{1}-\frac{64 K_{1}^{2}}{27 K_{2}} \frac{(2 a+2 b+3)(2 a+6 b+1)}{(2 a+3 b+2)^{2}} \\
& q=\frac{K_{2}}{\left(K_{1}-\lambda T\right) T}, \quad c=\frac{\left(K_{1}-\lambda T\right)^{2}}{K_{2}}
\end{aligned}
$$

Simplifications: background $(\lambda)$ is known estimated parameters are $c, q, a, b$

$$
\left\{\begin{array}{l}
\chi_{3}=\frac{\left(K_{1}-\lambda T\right) K_{3}}{K_{2}^{2}}=\frac{64(2 a+6 b+1)(2 a+2 b+3)}{27(2 a+3 b+2)^{2}} \\
\chi_{4}=\frac{\left(K_{1}-\lambda T\right)^{2} K_{4}}{K_{2}^{3}}=\frac{4(2 a+6 b+1)^{2}(4 a+3 b+8)}{(2 a+3 b+2)^{3}}
\end{array}\right.
$$

$$
\begin{gathered}
q=\frac{K_{2}}{\left(K_{1}-\lambda T\right) T}, \quad c=\frac{\left(K_{1}-\lambda T\right)^{2}}{K_{2}} \\
\text { In general } \quad \chi_{k}=\frac{\left(K_{1}-\lambda T\right)^{k-2} K_{k}}{K_{2}^{k-1}}
\end{gathered}
$$




\section{Solution of system 19}

Shape of the fourth order polynomial with respect to parameter a

$$
H_{1} a^{4}+H_{2} a^{3}+H_{3} a^{2}+H_{4} a+H_{5}=0
$$

$\alpha^{2} \beta^{2}-18 \alpha \beta+27 \alpha+16 \beta-27>0 \quad-$ red line $\alpha^{2} \beta^{2}-18 \alpha \beta+27 \alpha+16 \beta-27 \leq 0 \quad-$ blue line $\alpha=\frac{16875 K_{5} K_{3}}{16384 K_{4}^{2}} \quad \beta=\frac{1024 K_{4} K_{2}}{729 K_{3}^{2}}$

Root selection:

setting admissible ranges

$>$ minimization of $\chi^{2}$ criterion

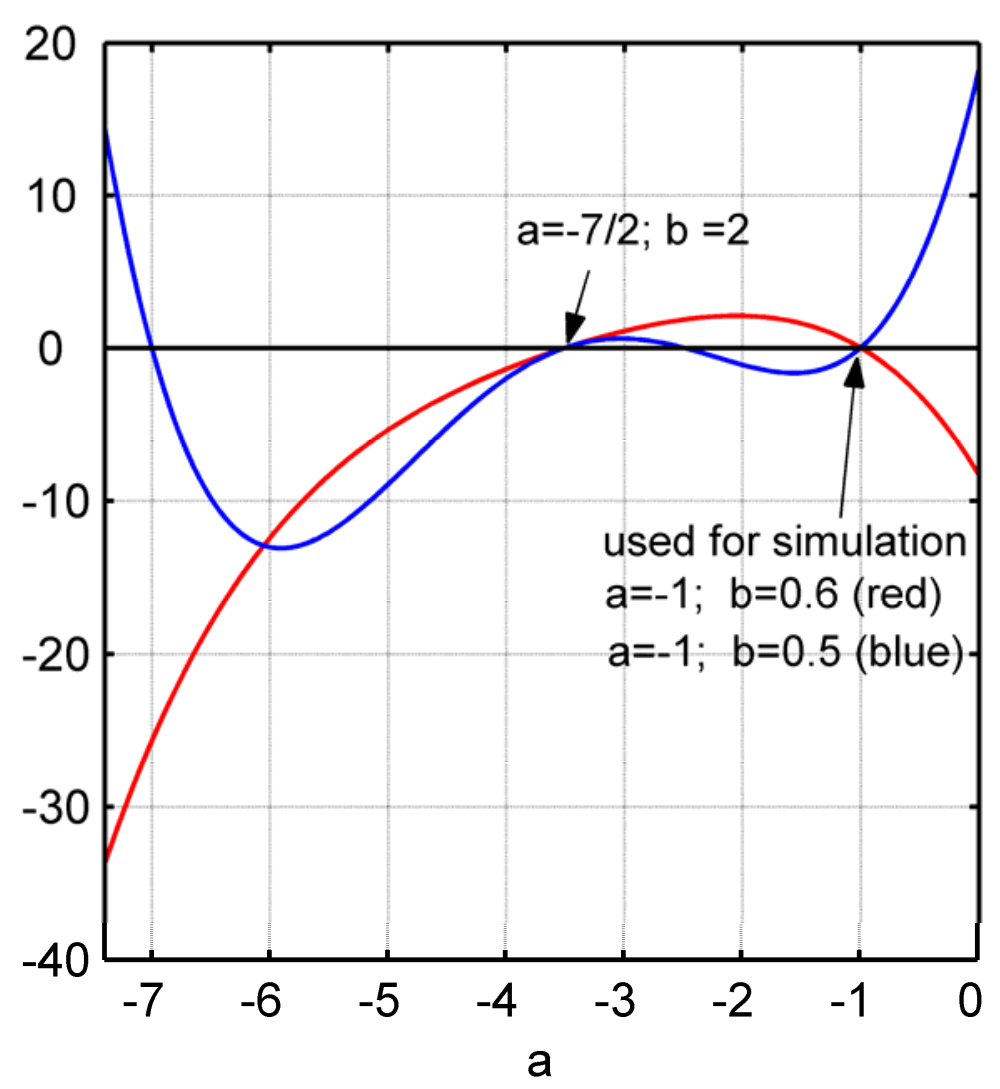

Fig. 1. Roots of polynomial 


\section{IG for two component model}

\section{Initial system of equations}

estimated parameters are $c_{1}, c_{2}, q_{1}, q_{2}, \lambda, a, b$

$K_{1}=\left(\lambda+c_{1} q_{1}+c_{2} q_{2}\right) T$

$K_{2}=\left(c_{1} q_{1}^{2}+c_{2} q_{2}^{2}\right) T^{2}$

$K_{3}=\left(c_{1} q_{1}^{3}+c_{2} q_{2}^{3}\right) T^{3} \frac{64(2 a+2 b+3)(2 a+6 b+1)}{27(2 a+3 b+2)^{2}}$

$K_{4}=\left(c_{1} q_{1}^{4}+c_{2} q_{2}^{4}\right) T^{4} \frac{4(4 a+3 b+8)(2 a+6 b+1)^{2}}{(2 a+3 b+2)^{3}}$

$K_{5}=\left(c_{1} q_{1}^{5}+c_{2} q_{2}^{5}\right) T^{5} \frac{4096(10 a+6 b+25)(2 a+6 b+1)^{3}}{625(2 a+3 b+2)^{4}}$

$K_{6}=\left(c_{1} q_{1}^{6}+c_{2} q_{2}^{6}\right) T^{6} \frac{4096(2 a+b+6)(2 a+6 b+1)^{4}}{27(2 a+3 b+2)^{5}}$

$K_{7}=\left(c_{1} q_{1}^{7}+c_{2} q_{2}^{7}\right) T^{7} \frac{8^{6}(14 a+6 b+49)(2 a+6 b+1)^{5}}{2401(2 a+3 b+2)^{6}}$

\section{Simplifications:}

1. background $(\lambda)$ and instrumental parameters $a$ and $b$ are known

$$
\begin{aligned}
& K_{1}-\lambda T=\left(c_{1} q_{1}+c_{2} q_{2}\right) T \\
& K_{2}=\left(c_{1} q_{1}^{2}+c_{2} q_{2}^{2}\right) T^{2} \\
& K_{3} / \chi_{3}=\left(c_{1} q_{1}^{3}+c_{2} q_{2}^{3}\right) T^{3} \\
& K_{4} / \chi_{4}=\left(c_{1} q_{1}^{4}+c_{2} q_{2}^{4}\right) T^{4}, \\
& \chi_{3}=\frac{64(2 a+6 b+1)(2 a+2 b+3)}{27(2 a+3 b+2)^{2}} \\
& \chi_{4}=\frac{4(2 a+6 b+1)^{2}(4 a+3 b+8)}{(2 a+3 b+2)^{3}}
\end{aligned}
$$

\section{2. background $(\lambda)$ is known}

A number of predefined parameters $a$ and b used for solution of the sysmem 25 . As result a number of sets of parameters is generated and the set resulting in lowest $\chi^{2}$ criterion is accepted. 


\begin{tabular}{|c|c|c|c|c|c|}
\hline & \multicolumn{5}{|c|}{$\begin{array}{l}\text { Testing of IG for one component model } \\
\text { on simulated data }\end{array}$} \\
\hline \multicolumn{6}{|c|}{ 1. IG for all parameters $c, q, \lambda, a, b$} \\
\hline \multicolumn{6}{|c|}{$\begin{array}{l}\text { Table 1. IG for one component model on noisy PCD at different } S / N \text {. IG were rejected when either } \lambda \text { was } \\
\text { negative or } a \text { and } b \text { exceed bounds }(-2,0) ;(0,2) \text { respectively. } T=5 \times 10^{-5} .50 \text { simulations in each series. }\end{array}$} \\
\hline \multirow[t]{2}{*}{ Parameter } & Used for & & Recovered & & \multirow{7}{*}{$\begin{array}{l}S / N=\sqrt{m p_{\max } /\left(1-p_{\max }\right)} \\
S / N_{\text {initial }}=\sqrt{m p_{\max }}= \\
\quad=\sqrt{\text { Value at Maximum }} \\
\text { here } p_{\max }=\max _{n}(P(n)), \\
m \text { is total number of photons }\end{array}$} \\
\hline & simulation & $S / N_{i}=7000$ & $S / N_{i}=3000$ & $S / N_{i}=1000$ & \\
\hline$c$ & 5 & $4.994 \pm 0.126$ & $4.882 \pm 0.205$ & $4.327 \pm 0.542$ & \\
\hline$q$ & 20000 & $20016 \pm 252$ & $20253 \pm 436$ & $21633 \pm 1462$ & \\
\hline$\lambda$ & 2000 & $2064 \pm 1260$ & $3208 \pm 2086$ & $9170 \pm 5948$ & \\
\hline$a$ & -1 & $-0.999 \pm 0.019$ & $-0.980 \pm 0.034$ & $-0.853 \pm 0.152$ & \\
\hline$b$ & 0.5 & $0.500 \pm 0.002$ & $0.499 \pm 0.003$ & $0.495 \pm 0.009$ & \\
\hline \multicolumn{6}{|c|}{ 2. IG for parameters $c, q, a, b$ ( $\lambda$ is known) } \\
\hline \multicolumn{6}{|c|}{ Table 2. IG calculated for one component model on noisy PCD at different S/N. $\lambda$ fixed to $2000 . T=5 \times 10^{-5}$. } \\
\hline \multirow[t]{2}{*}{ Parameter } & \multirow{2}{*}{$\begin{array}{l}\text { Used for } \\
\text { simulation }\end{array}$} & \multicolumn{3}{|c|}{ Recovered } & \\
\hline & & $S / N_{i}=1000$ & $S / N_{i}=300$ & $S / N_{i}=50$ & \\
\hline$c$ & 5 & $5.000 \pm 0.006$ & $4.999 \pm 0.025$ & $4.999 \pm 0.107$ & \\
\hline$q$ & 20000 & $20002 \pm 24$ & $20003 \pm 107$ & $20029 \pm 455$ & \\
\hline$a$ & -1 & $-1.000 \pm 0.004$ & $-1.006 \pm 0.015$ & $-1.048 \pm 0.106$ & \\
\hline$b$ & 0.5 & $0.500 \pm 0.008$ & $0.514 \pm 0.033$ & $0.522 \pm 0.183$ & 10 \\
\hline
\end{tabular}




\section{Testing of IG for two component model on simulated data}

IG for parameters $c_{1}, c_{2}, q_{1}, q_{2}(\lambda, a, b$ are known)

Table 3. IG calculated for two component model on noisy PCD at different $\mathrm{S} / \mathrm{N} . \lambda=1000 . a=-1 ; b=0.5$; $T=2 \times 10^{-5} .50$ simulations in each series.

\begin{tabular}{|c|l|l|l|}
\hline \multirow{2}{*}{ Parameter } & \multirow{2}{*}{$\begin{array}{c}\text { Used for } \\
\text { simulation }\end{array}$} & \multicolumn{2}{|c|}{ Recovered } \\
\cline { 3 - 4 } & & $S / N_{i}=1000$ & $S / N_{i}=100$ \\
\hline$c_{1}$ & 10 & $9.99 \pm 0.13$ & $10.11 \pm 1.70$ \\
\hline$q_{2}$ & 20000 & $19883 \pm 635$ & $18282 \pm 5013$ \\
\hline$c_{1}$ & 2 & $2.04 \pm 0.23$ & $2.59 \pm 1.64$ \\
\hline$q_{2}$ & 50000 & $49847 \pm 1404$ & $50931 \pm 11676$ \\
\hline
\end{tabular}




\begin{tabular}{|c|c|c|c|c|}
\hline & \multicolumn{3}{|c|}{$\begin{array}{c}\text { Testing of } \mathrm{IG} \text { for one component model } \\
\text { on measured data }\end{array}$} & $\begin{array}{ll}\mathrm{L} & \mathrm{U} \\
\mathrm{M} & \mathrm{C}\end{array}$ \\
\hline \multicolumn{5}{|c|}{ 1. IG for all parameters $c, q, \lambda, a, b$} \\
\hline \multicolumn{5}{|c|}{ Table 4. IG calculated for one component model on measured data (Alexa 488). $T=8 \times 10^{-6}$} \\
\hline Parameters & IG & Fit starting from IG & \multirow{7}{*}{$\begin{array}{l}\text { Confidential intervals are } \\
\text { calculated as Asymptotic } \\
\text { Standard Errors. }\end{array}$} & \\
\hline$\chi^{2}$ & 0.781 & 0.760 & & \\
\hline$c$ & 23.12 & $25.08 \pm 4.07$ & & \\
\hline$q$ & 19192 & $18426 \pm 1495$ & & \\
\hline$\lambda$ & 18424 & $27 \pm 37465$ & & \\
\hline$a$ & -4.41 & $-4.58 \pm 0.47$ & & \\
\hline$b$ & 3.22 & $3.47 \pm 0.63$ & & \\
\hline \multicolumn{5}{|c|}{ 2. IG for parameters $c, q, a, b$ ( $\lambda$ is estimated from additional measurement) } \\
\hline \multicolumn{5}{|c|}{ Table 5. IG calculated for one component model on measured data (Alexa 488). $\lambda$ fixed to $1500 . T=8 \times 10^{-6}$} \\
\hline Parameters & IG & Best fit $=$ fit starting from IG & \multirow{6}{*}{$\begin{array}{l}\text { Confidential intervals are } \\
\text { calculated as Asymptotic } \\
\text { Standard Errors. }\end{array}$} & \\
\hline$\chi^{2}$ & 0.668 & 0.666 & & \\
\hline$c$ & 24.916 & $24.923 \pm 0.189$ & & \\
\hline$q$ & 18500 & $18500 \pm 140.5$ & & \\
\hline$a$ & -1.463 & $-1.472 \pm 0.0014$ & & \\
\hline$b$ & 0.321 & $0.324 \pm 0.0004$ & & 12 \\
\hline
\end{tabular}




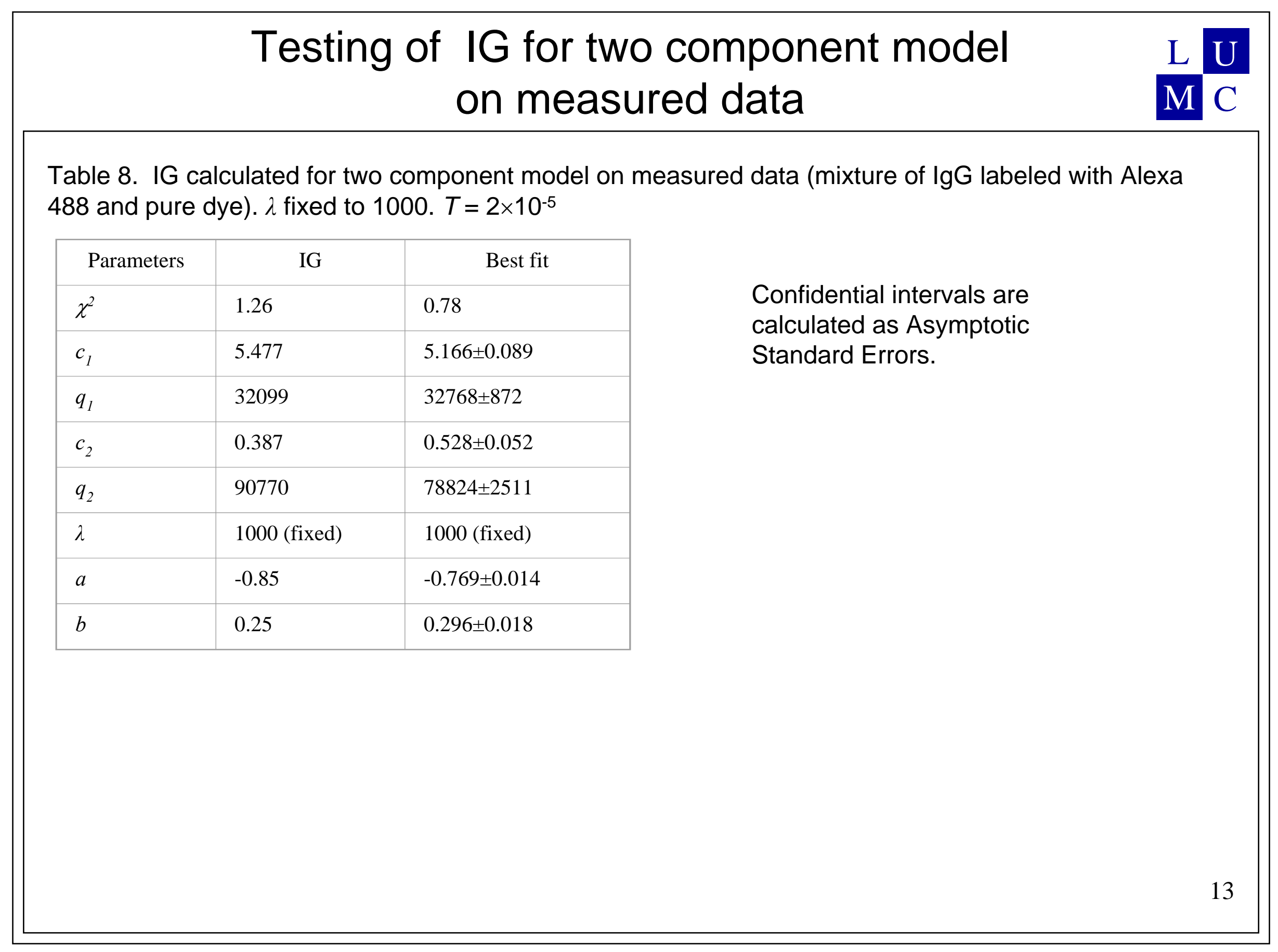




\section{minima in $\chi^{2}$ space}

Table 6. Parameters used for calculation (obtained from best fit of Alexa 488). $T=8 \times 10^{-6}$.

\begin{tabular}{|l|l|}
\hline Parameter & Value \\
\hline$c$ & 24.923 \\
\hline$q$ & 18500 \\
\hline$\lambda$ & 1500 \\
\hline$a$ & varied \\
\hline$b$ & varied \\
\hline
\end{tabular}

Table 7. Calculation of $\chi^{2}$ for all three roots of the system 22 .

\begin{tabular}{|l|l|l|l|}
\hline Parameter & set 1 & set 2 & set 3 \\
\hline$a$ & -1.393 & -1.463 & -4.643 \\
\hline$b$ & -0.861 & 0.321 & 3.539 \\
\hline$\chi^{2}$ & 0.732 & 0.668 & 0.711 \\
\hline
\end{tabular}

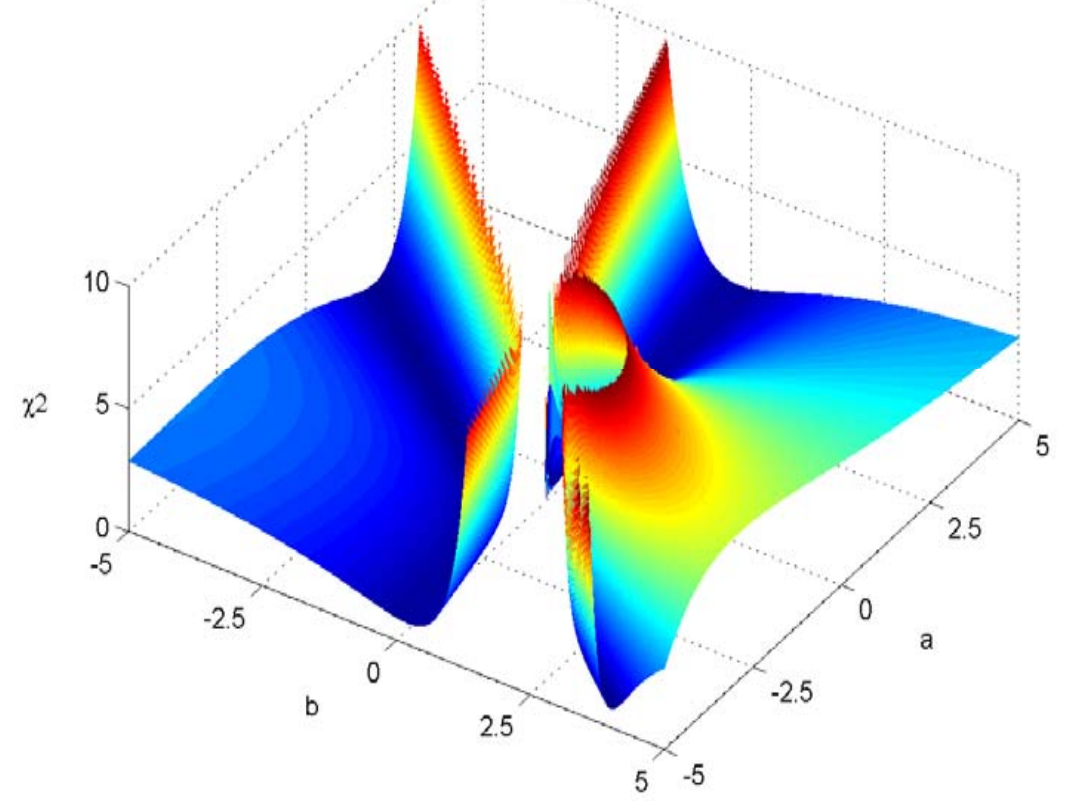

Fig. 2. $\chi^{2}$ surface plotted versus $a$ and $b$.

$$
\left\{\begin{array}{l}
\frac{64(2 a+6 b+1)(2 a+2 b+3)}{27(2 a+3 b+2)^{2}}=\frac{\left(K_{1}-\lambda T\right) K_{3}}{K_{2}^{2}}=\chi_{3} \\
\frac{4(2 a+6 b+1)^{2}(4 a+3 b+8)}{(2 a+3 b+2)^{3}}=\frac{\left(K_{1}-\lambda T\right)^{2} K_{4}}{K_{2}^{3}}=\chi_{4}
\end{array}\right.
$$




\section{Conclusions}

1. In theory, if we have molecular system with n-components, IG can be obtained as solution of system of equations 15.

2. A non iterative, straightforward method of IG calculation for one and two-component system is proposed.

3. Applicability of method was verified with testing on simulated and measured data.

4. A rule of selecting of IG from possible results is suggested.

5. Impact of possible discontinuity points in model domain and consequently in $\chi^{2}$ space is discussed

6. developed IG allows to increase the speed of analysis in most cases at least into 5 times. 